\title{
A comparative prognostic performance of definitions of Crohn-like lymphoid reaction in colorectal carcinoma
}

\author{
Younghoon Kim ${ }^{1,2,3}$, Jeong Mo Bae ${ }^{1,3}$, Jung Ho Kim ${ }^{1,3}$, Nam-Yun Cho ${ }^{1}$, Gyeong Hoon Kang ${ }^{1,2}$ \\ ${ }^{1}$ Laboratory of Epigenetics, Cancer Research Institute, Seoul National University College of Medicine, Seoul; \\ 2Department of Pathology, Seoul National University College of Medicine, Seoul; \\ ${ }^{3}$ Department of Pathology, Seoul National University Hospital, Seoul, Korea
}

\begin{abstract}
Background: The prognostic potential of Crohn-like lymphoid reaction (CLR) in colorectal carcinoma (CRC) has been investigated through the assessment of different criteria. Methods: The prognostic impact of CLR was investigated in 636 CRC patients to compare methods from previously published articles. These methods included CLR measured by number of lymphoid aggregates (LAs) (CLR count), LA size greater than or equal to $1 \mathrm{~mm}$ (CLR size), CLR density with a cutoff value of 0.38 , and subjective criteria as defined by intense CLR. Results: In univariate survival analysis, CLR-positive CRC as defined by the four aforementioned methods was associated with better overall survival (OS) (hazard ratio [HR], 0.463; 95\% confidence interval [Cl], 0.305 to $0.702 ; \mathrm{p}<.001 ; \mathrm{HR}, 0.656 ; 95 \% \mathrm{Cl}$, 0.411 to $1.046 ; p=.077 ; \mathrm{HR}, 0.363 ; 95 \% \mathrm{Cl}, 0.197$ to $0.669 ; p=.001$; and $\mathrm{HR}, 0.433 ; 95 \% \mathrm{Cl}, 0.271$ to $0.690 ; p<.001$, respectively) and disease-free survival (DFS) (HR, $0.411 ; 95 \% \mathrm{Cl}, 0.304$ to $0.639 ; p<.001 ; \mathrm{HR}, 0.528 ; 95 \% \mathrm{Cl}, 0.340$ to $0.821 ; \mathrm{p}=.004 ; \mathrm{HR}, 0.382 ; 95 \%$ $\mathrm{Cl}, 0.226$ to $0.645, p=.004$; and $\mathrm{HR}, 0.501 ; 95 \% \mathrm{Cl}, 0.339$ to $0.741 ; \mathrm{p}<.001$, respectively) than CLR-negative CRC, regardless of criteria with the exception of OS for CLR density. In multivariate analysis, two objective criteria (CLR count and CLR density) and one subjective criterion (intense CLR) for defining CLR were considered independent prognostic factors of OS and DFS in CRC patients. Conclusions: CLR has similar traits regardless of criteria, but CLR-positivity should be defined by objective criteria for better reproducibility and prognostic value.
\end{abstract}

Key Words: Colorectal neoplasms; Crohn-like lymphoid reaction; Histology; Prognosis

Received: August 24, 2020 Revised: September 21, 2020 Accepted: October 6, 2020

Corresponding Author: Gyeong Hoon Kang, MD, PhD, Department of Pathology, Seoul National University College of Medicine, 103 Daehak-ro, Jongro-gu, Seoul 03080, Korea Tel: +82-2-740-8273, Fax: +82-2-765-5600, E-mail: ghkang@snu.ac.kr

Colorectal cancer (CRC) is the fourth most common cause of cancer death worldwide, and its incidence is increasing in young patients [1]. Although CRC is a heterogeneous cancer with various subtypes, a lymphoid reaction is known to represent the immune response to the tumor [2-4]. Among these reactions, the Crohn-like lymphoid reaction (CLR) consists of lymphoid aggregates (LAs), with or without germinal centers that are distributed within the bowel wall, that surpass the invasion front (IF) of the tumor and are mostly localized in the muscularis propria and the pericolic adipose tissue [5]. Like other lymphoid reactions within CRC, CLR has not only been described as a means of antitumor immune response but also as a prognostic factor in several individual studies [4,6-8].

In $\mathrm{CRC}$, conditions that involve dense lymphocytic reactions, namely CLR, peritumoral reaction, intratumoral periglandular reaction, and tumor-infiltrating lymphocytes, are strongly associated with molecular subtypes microsatellite instability (MSI) and the CpG island methylator phenotype (CIMP) [9-13]. Inflammatory bowel diseases (IBD) including ulcerative colitis and Crohn's disease could also be the reason for CLR in patients with CRC. Since certain molecular subtypes or preceding IBD could be associated with the prognosis, it would be possible to assume that these factors may act as confounding factors between CLR and patient prognosis [14-16]. However, previous studies have found that CLR is a prognostic indicator even within MSI-high or colitis-associated CRC populations [6,17]. Therefore, it has been acknowledged that a quantifying immune reaction could benefit in providing additional survival information in overall CRC patients [18].

Several different histological assessment systems have been 
developed to determine the correlation between CLR and prognosis. Some of the criteria for identifying positive CLR have been categorized and applied to a single MSI-high CRC patient cohort [4-7]. However, a comparative analysis that assesses alternative criteria in a single CRC population regardless of molecular subtype or preceding disease conditions has been unavailable up to this point. In this study, we reviewed previous studies to investigate the methods that were used to measure CLR in CRC and categorized the criteria being used. Furthermore, we compared each criterion in a single CRC patient set to characterize different aspects of various CLR criteria including association with prognosis, age, sex, molecular subtype, and other histological characteristics. We attempted to clarify the prognostic value of CLR in CRC according to various assessment systems.

\section{MATERIALS AND METHODS}

\section{Patients and specimens}

We retrospectively analyzed 767 CRC patients who underwent tumor resection between January 2004 and December 2006. Among them, 636 patients whose whole section slide samples were available from the pathology archive at Seoul National University Hospital were selected. The available tissue samples were formalin-fixed, paraffin-embedded tissues that were obtained from CRC specimens resected during curative surgery. Patients exposed to chemotherapy or radiotherapy before resection were excluded. Patient information including age, sex, and other clinical or pathological data were collected from the electronic medical records.

\section{Analysis of molecular subtypes and KRAS and BRAF mutation status}

MSI status was determined using five microsatellite markers (BAT25, BAT26, D2S123, D5S345, and D17S250). MSI-high was defined when instability was present in $\geq 40 \%$ of the mark- ers. MSI-low and microsatellite stable (MSS) were defined as instability in one marker and none of the markers, respectively. CIMP status was determined by quantification of the DNA methylation levels in eight markers (CACNA1G, CDKN2A, CRABP1, IGF2, MLH1, NEUROG1, RUNX3, and SOCS1) via bisulfate DNA modification and MethyLight as described previously [19]. CIMP was considered positive when more than four markers were methylated but negative otherwise. To evaluate mutations in KRAS and BRAF, microdissected tissues were manually collected and incubated in a mixture of lysis buffer and proteinase $\mathrm{K}$ at $55^{\circ} \mathrm{C}$ for 2 days. KRAS mutations were identified by direct sequencing of codons 12 and 13. BRAF mutations were identified by allele-specific polymerase chain reaction in codon 600 as described previously [20].

\section{Pathological assessment of CLR}

Two pathologists (YK and JMB) assessed the CLR status of 636 CRC patient slides. Criteria used for assessments were obtained from the review of previous studies (Table 1) [4,6,7,9, 17,21-23]. These criteria include (1) counting the number of three or more LAs, from Buckowitz et al. (Fig. 1A, CLR count), (2) size-based assessment of $\mathrm{LA} \geq 1 \mathrm{~mm}$, from Ueno et al. (Fig. 1B, CLR size), (3) criteria from the Väyrynen-Mäkinen group, which considers a density of CLR (the number of LAs divided by the length of the IF) greater than or equal to 0.38 to be high (Fig. 1C, CLR density), and (4) intense CLR (Fig. 1D), which represents less objective criteria such as transmural and intense CLRs $[4,7,21]$.

\section{Statistical analysis}

R (ver. 3.3.1, R Foundation for Statistical Computing, Vienna, Austria) was used to analyze the CRC patient samples. To compare categorical variables, Fisher exact test was performed. For the univariate analysis, Kaplan-Meier analysis with a log-rank test was used to investigate the association between clinicopath-

Table 1. Characteristics of studies of the correlation between CLR and survival

\begin{tabular}{lccccll}
\hline Study & Stage & No. & Positive (\%) & Subtype & Outcome & CLR criteria \\
\hline Buckowitz et al. (2005) [21] & I-IV & 118 & 35.6 & - & OS & $\geq 3$ LAs \\
Ogino et al. (2009) [9] & I-IV & 843 & 27.3 & - & CSS, OS & Transmural CLR \\
Kim et al. (2015) [6] & I-IV & 212 & $21.5-65.1$ & MSI-H & DFS & Intense CLR, LA $\geq 1 \mathrm{~mm}$, LA/IF $\geq 0.38$ \\
Rozek et al. (2016) [22] & I-IV & 1484 & 47.1 & & CSS, OS & $\geq 3$ LAs \\
Kakar et al. (2004) [23] & I-IV & 248 & 41.1 & Mucinous & OS & Intense CLR \\
Lewis et al. (2013) [17] & I-IV & 89 & 56.2 & Colitis-associated & PFS, OS & Transmural CLR \\
Ueno et al. (2013) [4] & I-IV & 1354 & 73.3 & - & DFS & LA $\geq 1$ mm \\
Vayrynen et al. (2014) [7] & I-IV & 567 & - & - & CSS & LA/IF $\geq 0.38$ \\
\hline
\end{tabular}

CLR, Crohn-like lymphoid reaction; OS, overall survival; LA, lymphoid aggregate; CSS, cancer-specific survival; MSI-H, microsatellite instability-high; DFS, disease-free survival; IF, invasion front; PFS, progression-free survival. 

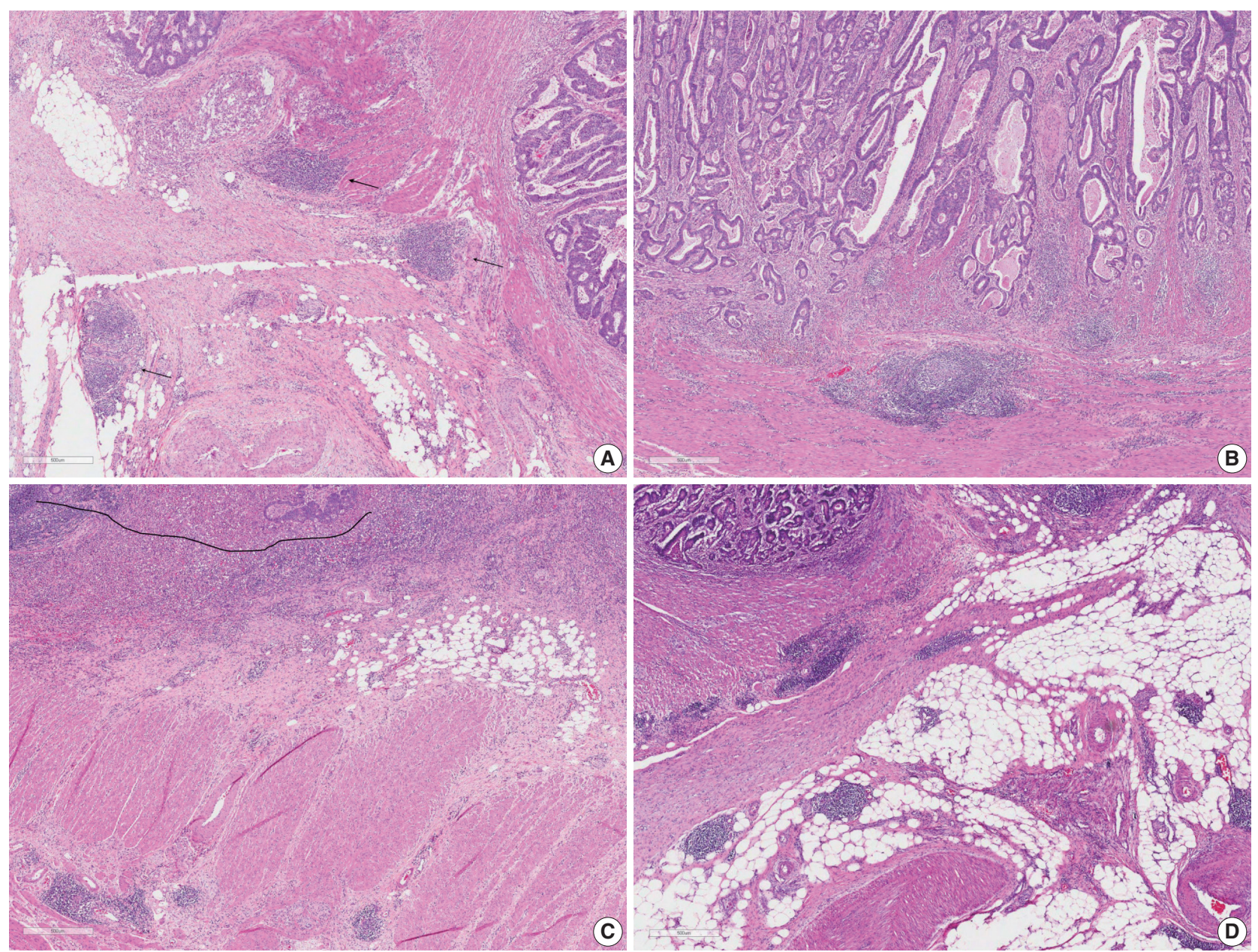

Fig. 1. Examples of Crohn-like lymphoid reaction (CLR)-positive cases according to each criterion. (A) At least three lymphoid aggregates (LAs) (arrow), positive according to CLR count. (B) A single LA, which is greater than or equal to $1 \mathrm{~mm}$ in diameter, positive by CLR size. (C) The number of LAs divided by the length of the invasion front (bold line) is greater than or equal to 0.38 (positive according to CLR density). (D) An intense LA in the proper muscle and subserosa.

ological parameters including CLR status and survival. Multivariate survival analysis, which was performed when the p-value was less than .05 in the univariate analysis for a given parameter, was conducted using the Cox proportional hazards regression model.

\section{RESULTS}

\section{Association between clinicopathological parameters and CLR in CRC patients}

The clinicopathological features of CRCs with CLR-positivity according to different CLR criteria are summarized in Table 2. CLR-positive CRCs, regardless of the CLR criteria used, were significantly associated with a lower pTNM stage. Lymphatic invasion demonstrated a significant inverse correlation with CLRpositive CRCs as defined by CLR count, CLR size, and intense
CLR ( $\mathrm{p}=.002, \mathrm{p}=.005$, and $\mathrm{p}=.021$, respectively). CLR-positivity was positively correlated with MSI-high and demonstrated preference for the colon over the rectum and for the right-sided colon over the left-sided colon as the site of origin, regardless of criteria.

\section{Prognostic value of CLR according to different criteria in CRC patients}

Univariate survival analysis revealed that CLR-positive CRC is associated with significantly better overall survival (OS) (hazard ratio $[\mathrm{HR}], 0.463 ; 95 \%$ confidence interval $[\mathrm{CI}], 0.305$ to 0.702 ; $\mathrm{p}<.001 ; \mathrm{HR}, 0.363 ; 95 \% \mathrm{CI}, 0.197$ to $0.669 ; \mathrm{p}=.001$; and HR, $0.433 ; 95 \% \mathrm{CI}, 0.271$ to $0.690 ; \mathrm{p}<.001$, respectively) and disease-free survival (DFS) (HR, 0.411; 95\% CI, 0.304 to 0.639 ; $\mathrm{p}<.001 ; \mathrm{HR}, 0.382 ; 95 \% \mathrm{CI}, 0.226$ to $0.645 ; \mathrm{p}=.004$; and HR, $0.501 ; 95 \% \mathrm{CI}, 0.339$ to $0.741 ; \mathrm{p}<.001$, respectively) than 
Table 2. Clinicopathological parameters and CLR

\begin{tabular}{|c|c|c|c|c|c|c|c|c|c|}
\hline Variable & & CLR count (\%) & $p$-value & CLR size (\%) & $p$-value & CLR density (\%) & $p$-value & Intense CLR (\%) & $\mathrm{p}$-value \\
\hline Total & 636 & $169(26.6)$ & & $104(16.4)$ & & $94(14.8)$ & & $140(22.0)$ & \\
\hline Age & & & .470 & & .236 & & .736 & & .847 \\
\hline$\geq 65$ & 358 & $91(25.4)$ & & $53(14.8)$ & & $51(14.2)$ & & 80 (22.3) & \\
\hline$<65$ & 278 & 78 (28.1) & & $51(18.4)$ & & $43(15.5)$ & & $60(21.6)$ & \\
\hline Sex & & & .583 & & .827 & & .670 & & .626 \\
\hline Male & 383 & $105(27.4)$ & & $64(16.7)$ & & $65(17.0)$ & & $87(22.7)$ & \\
\hline Female & 253 & 64 (25.3) & & $40(15.9)$ & & $29(11.5)$ & & $53(20.9)$ & \\
\hline pTNM stage & & & .001 & & .041 & & .002 & & .005 \\
\hline | or || & 306 & $100(32.7)$ & & 60 (19.7) & & 59 (19.3) & & $82(26.8)$ & \\
\hline III or IV & 330 & 69 (20.9) & & $44(13.4)$ & & 35 (10.6) & & 58 (17.6) & \\
\hline Lymphatic invasion & & & .002 & & .131 & & .005 & & .021 \\
\hline Absent & 353 & $111(31.4)$ & & 65 (18.5) & & 65 (18.4) & & $90(25.5)$ & \\
\hline Present & 283 & $58(20.5)$ & & 39 (13.8) & & 29 (10.2) & & $50(17.7)$ & \\
\hline Vascular invasion & & & .118 & & .214 & & .072 & & .486 \\
\hline Absent & 549 & $152(27.7)$ & & $94(17.2)$ & & 87 (15.8) & & $124(22.6)$ & \\
\hline Present & 87 & 17 (19.5) & & $10(11.5)$ & & $7(8.0)$ & & $16(18.4)$ & \\
\hline Perineural invasion & & & .141 & & .102 & & .065 & & .178 \\
\hline Absent & 485 & $136(28.0)$ & & $86(17.8)$ & & 79 (16.3) & & $113(23.3)$ & \\
\hline Present & 151 & $33(21.9)$ & & $18(11.9)$ & & $15(9.9)$ & & 27 (17.9) & \\
\hline MSI status & & & .002 & & .002 & & .001 & & .006 \\
\hline Low/stable & 581 & $144(24.8)$ & & $86(14.8)$ & & 77 (13.3) & & $119(20.5)$ & \\
\hline High & 55 & $25(45.5)$ & & $18(33.3)$ & & $17(30.9)$ & & 21 (38.2) & \\
\hline CIMP & & & .274 & & .510 & & .365 & & .438 \\
\hline Low & 595 & $155(26.1)$ & & 96 (16.2) & & $86(14.5)$ & & $129(21.7)$ & \\
\hline High & 41 & 14 (34.1) & & $8(20.0)$ & & $8(19.5)$ & & $11(26.8)$ & \\
\hline KRAS mutation & & & .839 & & .466 & & .704 & & $>.99$ \\
\hline Absent & 470 & $124(26.4)$ & & 74 (15.8) & & 68 (14.5) & & $104(22.1)$ & \\
\hline Present & 166 & $45(27.1)$ & & 30 (18.2) & & $26(15.7)$ & & $36(21.7)$ & \\
\hline BRAF mutation & & & .429 & & .813 & & .619 & & .290 \\
\hline Absent & 602 & 158 (26.2) & & 98 (16.3) & & $88(14.6)$ & & $130(21.6)$ & \\
\hline Present & 34 & $11(32.4)$ & & $6(17.6)$ & & $6(17.6)$ & & $10(29.4)$ & \\
\hline Tumor location & & & .003 & & .001 & & .005 & & .005 \\
\hline Colon & 451 & $135(29.9)$ & & 87 (19.4) & & $78(17.3)$ & & 78 (17.3) & \\
\hline Rectum & 185 & $34(18.4)$ & & $17(9.2)$ & & $16(8.6)$ & & $16(8.6)$ & \\
\hline Tumor side & & & .005 & & .009 & & .002 & & .045 \\
\hline Left & 461 & $60(9.4)$ & & $39(6.1)$ & & $38(6.0)$ & & $47(7.4)$ & \\
\hline Right & 175 & 109 (17.1) & & 65 (10.2) & & $56(8.8)$ & & $93(14.6)$ & \\
\hline
\end{tabular}

CLR, Crohn-like lymphoid reaction; MSI, microsatellite instability; CIMP, CpG island methylator phenotype.

CLR-negative CRC, when the criteria of CLR count, CLR density, and intense CLR were applied (Fig. 2). When CLR was assessed with the size criteria, it was significantly correlated only with improved DFS (HR, 0.528; 95\% CI, 0.340 to 0.821; $\mathrm{p}=.004)$, but not with better OS (HR, 0.656; $95 \%$ CI, 0.411 to $1.046 ; \mathrm{p}=$ .077). The correlation between survival and CLR showed identical significance when evaluating only MSI-low and MSS CRC patients (Supplementary Table S1). In the multivariate survival analysis, CLR-positivity based on CLR count, CLR density, and intense CLR were determined to be an independent prognostic factors after adjustment for lymphatic invasion, perineural invasion, venous invasion, and pTNM stage (Table 3). When ex- cluding MSI-high CRC, CLR count and intense CLR were significantly associated with survival (Supplementary Table S2).

\section{DISCUSSION}

Strong lymphocytic reactions have been suspected to predict favorable prognoses in CRC. Among the four entities of CLR, peritumoral reaction, intratumoral periglandular reaction, and tumor-infiltrating lymphocyte (TIL), TIL has been the target of most interest and the front-runner when evaluating the lymphatic reaction of CRC [13,24-28]. Therefore, the correlation between TIL and survival has been established in multiple large- 

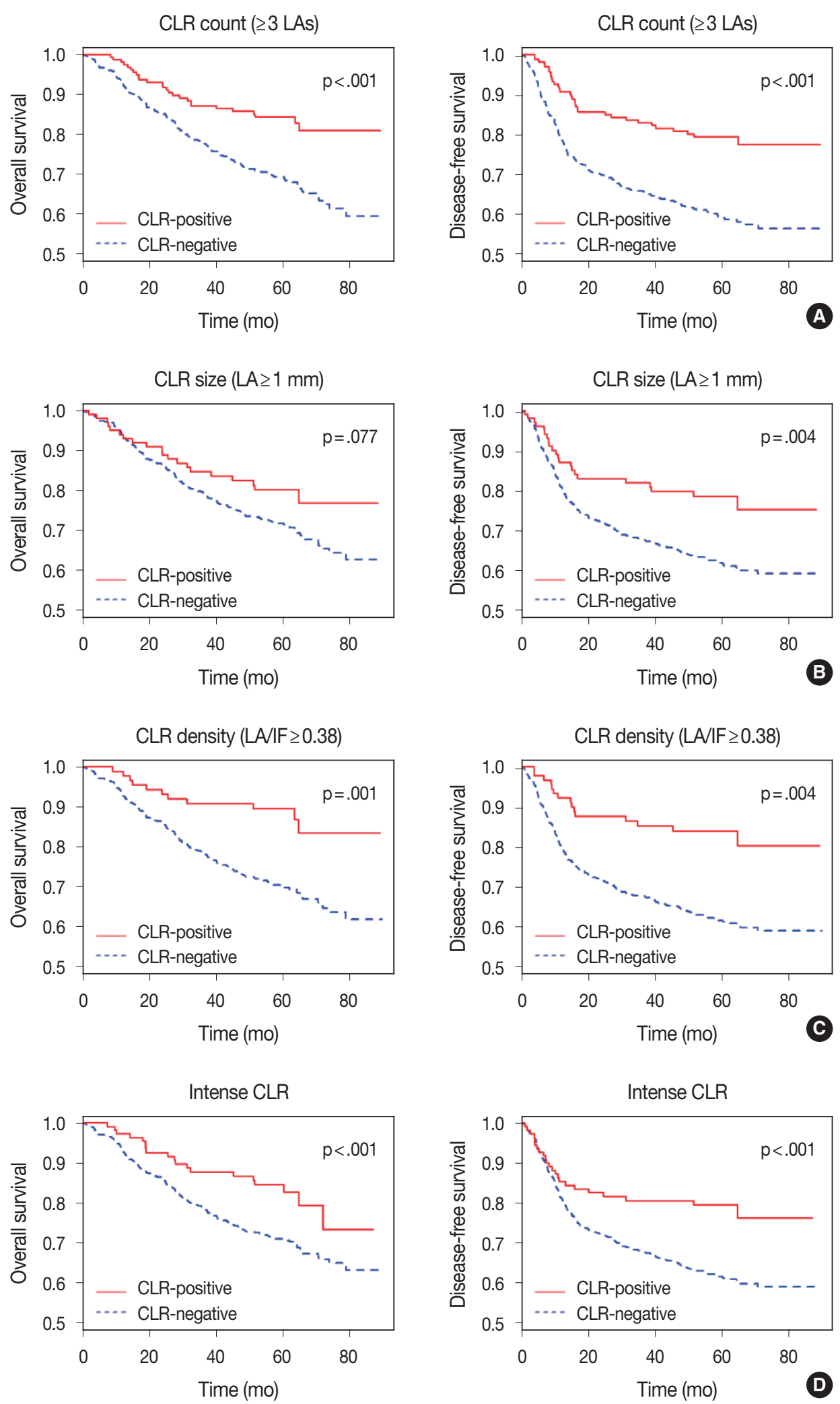

Fig. 2. Correlations between each Crohn-like lymphoid reaction (CLR) criterion and survival (overall survival and disease-free survival). (A) Kaplan-Meier curves for CLR count (at least three lymphoid aggregates [LAs]). (B) Kaplan-Meier curves for CLR size (LA $\geq 1 \mathrm{~mm}$ in diameter). (C) Kaplan-Meier curves for CLR density (the number of LAs divided by the length of the invasion front $\geq 0.38$ ). (D) Kaplan-Meier curves for intense CLR. 
Table 3. Multivariate analysis of CLR according to Cox proportional hazards regression model

\begin{tabular}{|c|c|c|c|c|c|c|}
\hline \multirow{2}{*}{ Criteria } & \multicolumn{3}{|c|}{ Overall survival } & \multicolumn{3}{|c|}{ Disease-free survival } \\
\hline & $\mathrm{HR}$ & $95 \% \mathrm{Cl}$ & $p$-value & $\mathrm{HR}$ & $95 \% \mathrm{Cl}$ & $p$-value \\
\hline CLR count & 0.595 & $0.390-0.907$ & .016 & 0.559 & $0.384-0.813$ & .002 \\
\hline CLR size & & & & 0.687 & $0.440-1.072$ & .098 \\
\hline CLR density & 0.519 & $0.280-0.962$ & .037 & 0.558 & $0.328-0.949$ & .031 \\
\hline Intense CLR & 0.610 & $0.378-0.984$ & .043 & 0.610 & $0.395-0.941$ & .025 \\
\hline
\end{tabular}

Each variable was adjusted for lymphatic, perineural, and venous invasion and pTNM stage. One of the CLR criteria was included for each model. $\mathrm{CLR}$, Crohn-like lymphoid reaction; $\mathrm{HR}$, hazard ratio; $\mathrm{Cl}$, confidence interval.

scale studies $[8,29,30]$. Studies suggest that TIL should be considered as a superior predicting prognostic factor to other molecular markers, including MSI and the KRAS and BRAF mutations, and to the consensus molecular subtype from the CRC subtype consortium [8]. However, TIL has disadvantages when clinical applications are considered. Since a standardized area of interest has not been established, studies range from counting TILs in high power fields and single tissue microarray cores to whole slide sections [31,32]. Moreover, intratumoral epithelial and stromal regions, as well as subtypes of TILs according to immunohistochemical data, could also have differential or opposing influence on survival [33]. Unlike TIL, CLR has been uniformly addressed with whole section slides and could be considered a more reliable marker for evaluating the prognostic potential of the lymphatic reaction in CRC.

The role of CLR in CRC can be regarded as a tertiary lymphoid structure that is associated with the host immune response against tumor cells [4]. While some studies have reported CLR as a prognostic factor, the results are not always in agreement $[6,9]$. Furthermore, various methods used to evaluate CLR-positivity have been developed, but the effects of different assessment methods have not been completely elucidated and need to be reviewed in detail. In this study, we observed different aspects of CLR and survival by applying multiple criteria to a single cohort of CRC patients for the comparison of each criterion. According to univariate analysis, CLR was prognostic with any of the four criteria, but not all criteria could demonstrate CLR as an independent prognostic factor in multivariate analysis. However, we have previously reported that interobserver reproducibility is higher with objective criteria than with subjective criteria [6]. Therefore, in the present study, we defined CLR as positive when at least 3 LAs were present and when LA/IF greater than or equal to 0.38 was observed.

In this study, we limited our focus to criteria that dichotomized the CRC patients according to CLR, and therefore, semiquantitative three-tier grading by Graham and Appelman [5] was not included. Nevertheless, the findings from the previous studies provided the basis of the criteria for this comparative analysis.

We clarified assessment methods for CLRs according to those presented in previous studies, analyzed $636 \mathrm{CRC}$ cases for CLR according to four different criteria, and correlated CLR with clinicopathological features and prognoses. Findings of our study suggest that CLR should be considered as an independent prognostic marker, and for clinical practice, CLR-positivity should be defined with objective criteria such as at least 3 LAs or LA/IF greater than or equal to 0.38 .

\section{Supplementary Information}

The Data Supplement is available with this article at https://doi.org/10.4132/ jptm.2020.10.06.

\section{Ethics Statement}

This study was approved by the Ethics Committee of the Seoul National University Hospital, which waived the requirement to obtain informed consent (H-1313-050-542).

\section{ORCID}

Younghoon Kim https://orcid.org/0000-0003-4656-0188 Jeong Mo Bae https://orcid.org/0000-0003-0462-3072 Jung Ho Kim https://orcid.org/0000-0002-6031-3629 Nam-Yun Cho https://orcid.org/0000-0002-5736-2956 Gyeong Hoon Kang https://orcid.org/0000-0003-2380-6675

\section{Author Contributions}

Conceptualization: GHK. Data curation: YK, JMB. Formal analysis: YK, NYC. Funding acquisition: GHK. Investigation: YK, NYC. Methodology: YK. Resources: JMB. Software: YK. Supervision: GHK. Validation: GHK. Visualization: YK. Writing_original draft: YK. Writing_review \& editing: JHK, GHK. Approval of final manuscript: all authors.

\section{Conflicts of Interest}

GHK and JHK, contributing editors of the Journal of Pathology and Translational Medicine, were not involved in the editorial evaluation or decision to publish this article. All remaining authors have declared no conflicts of interest.

\section{Funding Statement}

This work was supported by grants from the National Research Foundation (NRF) funded by the Korean Ministry of Science, ICT and Future Planning (2011-0030049 and 2016M3A9B6026921), a grant from the Korea Health Technology R\&D Project through the Korea Health Industry 
Development Institute funded by the Korean Ministry of Health and Welfare (HI14C1277), and a grant from the SNUH Research Fund (2320160090 (2016-1999))

\section{References}

1. Siegel RL, Miller KD, Jemal A. Cancer statistics, 2017. CA Cancer J Clin 2017; 67: 7-30.

2. Donehower LA, Creighton CJ, Schultz N, et al. MLH1-silenced and non-silenced subgroups of hypermutated colorectal carcinomas have distinct mutational landscapes. J Pathol 2013; 229: 99-110.

3. Guinney J, Dienstmann R, Wang X, et al. The consensus molecular subtypes of colorectal cancer. Nat Med 2015; 21: 1350-6.

4. Ueno H, Hashiguchi Y, Shimazaki H, et al. Objective criteria for crohn-like lymphoid reaction in colorectal cancer. Am J Clin Pathol 2013; 139: 434-41.

5. Graham DM, Appelman HD. Crohn's-like lymphoid reaction and colorectal carcinoma: a potential histologic prognosticator. Mod Pathol 1990; 3: 332-5.

6. Kim JH, Kim KJ, Bae JM, et al. Comparative validation of assessment criteria for Crohn-like lymphoid reaction in colorectal carcinoma. J Clin Pathol 2015; 68: 22-8.

7. Vayrynen JP, Sajanti SA, Klintrup K, et al. Characteristics and significance of colorectal cancer associated lymphoid reaction. Int J Cancer 2014; 134: 2126-35.

8. Williams DS, Mouradov D, Jorissen RN, et al. Lymphocytic response to tumour and deficient DNA mismatch repair identify subtypes of stage II/III colorectal cancer associated with patient outcomes. Gut 2019; 68: 465-74.

9. Ogino S, Nosho K, Irahara N, et al. Lymphocytic reaction to colorectal cancer is associated with longer survival, independent of lymph node count, microsatellite instability, and $\mathrm{CpG}$ island methylator phenotype. Clin Cancer Res 2009; 15: 6412-20.

10. Jass JR. HNPCC and sporadic MSI-H colorectal cancer: a review of the morphological similarities and differences. Fam Cancer 2004; 3: 93-100.

11. Alexander J, Watanabe T, Wu TT, Rashid A, Li S, Hamilton SR. Histopathological identification of colon cancer with microsatellite instability. Am J Pathol 2001; 158: 527-35.

12. Jass JR. Classification of colorectal cancer based on correlation of clinical, morphological and molecular features. Histopathology 2007; 50: 113-30.

13. Galon J, Costes A, Sanchez-Cabo F, et al. Type, density, and location of immune cells within human colorectal tumors predict clinical outcome. Science 2006; 313: 1960-4.

14. Juo YY, Johnston FM, Zhang DY, et al. Prognostic value of CpG island methylator phenotype among colorectal cancer patients: a systematic review and meta-analysis. Ann Oncol 2014; 25: 2314-27.

15. Ording AG, Horvath-Puho E, Erichsen R, et al. Five-year mortality in colorectal cancer patients with ulcerative colitis or Crohn's disease: a nationwide population-based cohort study. Inflamm Bowel Dis 2013; 19: 800-5.

16. Guastadisegni C, Colafranceschi M, Ottini L, Dogliotti E. Microsatellite instability as a marker of prognosis and response to therapy: a meta-analysis of colorectal cancer survival data. Eur J Cancer 2010; 46: 2788-98.

17. Lewis B, Lin J, Wu X, et al. Crohn's disease-like reaction predicts fa- vorable prognosis in colitis-associated colorectal cancer. Inflamm Bowel Dis 2013; 19: 2190-8.

18. Ferris RL, Galon J. Additional support for the introduction of immune cell quantification in colorectal cancer classification. J Natl Cancer Inst 2016; 108: djw033.

19. Bae JM, Kim JH, Rhee YY, Cho NY, Kim TY, Kang GH. Annexin A10 expression in colorectal cancers with emphasis on the serrated neoplasia pathway. World J Gastroenterol 2015; 21: 9749-57.

20. Lee DW, Kim KJ, Han SW, et al. KRAS mutation is associated with worse prognosis in stage III or high-risk stage II colon cancer patients treated with adjuvant FOLFOX. Ann Surg Oncol 2015; 22: $187-94$.

21. Buckowitz A, Knaebel HP, Benner A, et al. Microsatellite instability in colorectal cancer is associated with local lymphocyte infiltration and low frequency of distant metastases. Br J Cancer 2005; 92: 1746-53.

22. Rozek LS, Schmit SL, Greenson JK, et al. Tumor-infiltrating lymphocytes, Crohn's-like lymphoid reaction, and survival from colorectal cancer. J Natl Cancer Inst 2016; 108: djw027.

23. Kakar S, Aksoy S, Burgart LJ, Smyrk TC. Mucinous carcinoma of the colon: correlation of loss of mismatch repair enzymes with clinicopathologic features and survival. Mod Pathol 2004; 17: 696-700.

24. Titu LV, Monson JR, Greenman J. The role of CD8(+) T cells in immune responses to colorectal cancer. Cancer Immunol Immunother 2002; 51: 235-47.

25. Ling A, Edin S, Wikberg ML, Oberg A, Palmqvist R. The intratumoural subsite and relation of CD8(+) and FOXP3(+) T lymphocytes in colorectal cancer provide important prognostic clues. Br J Cancer 2014; 110: 2551-9.

26. Pages F, Berger A, Camus M, et al. Effector memory T cells, early metastasis, and survival in colorectal cancer. N Engl J Med 2005; 353: 2654-66.

27. Mei Z, Liu Y, Liu C, et al. Tumour-infiltrating inflammation and prognosis in colorectal cancer: systematic review and meta-analysis. Br J Cancer 2014; 110: 1595-605.

28. Kim Y, Bae JM, Li G, Cho NY, Kang GH. Image analyzer-based assessment of tumor-infiltrating $\mathrm{T}$ cell subsets and their prognostic values in colorectal carcinomas. PLoS One 2015; 10: e0122183.

29. Nosho K, Baba Y, Tanaka N, et al. Tumour-infiltrating T-cell subsets, molecular changes in colorectal cancer, and prognosis: cohort study and literature review. J Pathol 2010; 222: 350-66.

30. Frey DM, Droeser RA, Viehl CT, et al. High frequency of tumorinfiltrating FOXP3(+) regulatory $\mathrm{T}$ cells predicts improved survival in mismatch repair-proficient colorectal cancer patients. Int J Cancer 2010; 126: 2635-43.

31. Turksma AW, Coupe VM, Shamier MC, et al. Extent and location of tumor-infiltrating lymphocytes in microsatellite-stable colon cancer predict outcome to adjuvant active specific immunotherapy. Clin Cancer Res 2016; 22: 346-56.

32. Li Y, Liang L, Dai W, et al. Prognostic impact of programed cell death-1 (PD-1) and PD-ligand 1 (PD-L1) expression in cancer cells and tumor infiltrating lymphocytes in colorectal cancer. Mol Cancer 2016; 15: 55.

33. Jakubowska K, Kisielewski W, Kanczuga-Koda L, Koda M, Famulski W. Stromal and intraepithelial tumor-infiltrating lymphocytes in colorectal carcinoma. Oncol Lett 2017; 14: 6421-32. 